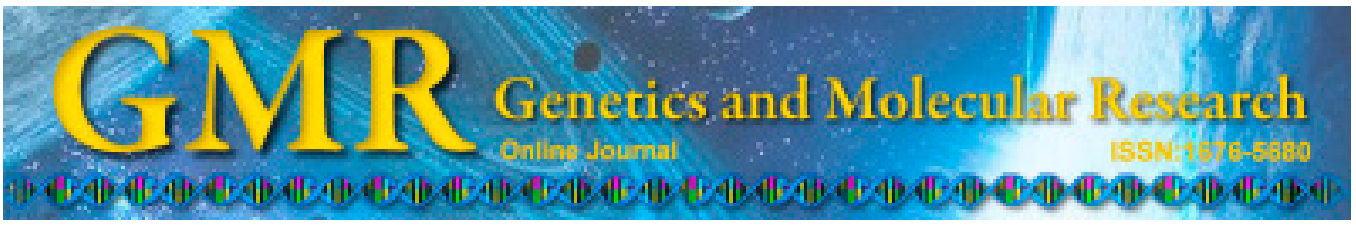

Case Report

\title{
Diffuse large B-cell lymphoma in the primary bone marrow
}

\author{
Y. Hu, S.L. Chen, Z.X. Huang, W. Gao and N. An \\ Department of Hematology, Beijing Chaoyang Hospital Affiliated to Capital \\ Medical University, Beijing, China \\ Corresponding author: $\mathrm{Y}$. Hu \\ E-mail: yinghucn@163.com
}

Genet. Mol. Res. 14 (2): 6247-6250 (2015)

Received August 20, 2014

Accepted January 21, 2015

Published June 9, 2015

DOI http://dx.doi.org/10.4238/2015.June.9.10

\begin{abstract}
This study aimed to improve understanding of the diagnosis, treatment, and prognosis of primary bone marrow (PBM) diffuse large B-cell lymphoma (DLBCL), a rare illness. We report a 56-year-old man with pancytopenia and hyperbilirubinemia but without lymphadenopathy, hepatomegaly, or splenomegaly. Bone marrow aspiration, flow cytometry, biopsy, and immunohistochemistry confirmed DLBCL. Two cycles of rituximab, cyclophosphamide, doxorubicin, vincristine, and prednisone were administered. Blood cell numbers and hyperbilirubinemia improved. Although the patient did not completely recover, he survived for at least 3 years after chemotherapy and receiving blood transfusions. PBM DLBCL is a distinct, aggressive lymphoma characterized by lymphoma cells only in the bone marrow and effectively treated via chemotherapy. Prognoses for PBM DLBCL vary.
\end{abstract}

Key words: Bone marrow; Diffuse large B-cell lymphoma 


\section{INTRODUCTION}

Diffuse large B-cell lymphoma (DLBCL) is a heterogeneous subtype of lymphoma. It is histologically characterized by the presence of large B-cells in tissue sections with various B-cell markers and high proliferation indices, in keeping with the aggressive nature of the disease. As with other subtypes of lymphoma, a risk stratification approach based on the International Prognostic Index (IPI) is used to predict prognosis for DLBCL. Large cell lymphoma with primary bone marrow (PBM) involvement is exceptional, although secondary involvement is sometimes observed. In this article, we describe the clinical and histologic characteristics of PBM DLBCL.

\section{CASE REPORT}

A 56-year-old man presenting with fatigue, pale skin, and yellow sclera was referred to our hospital in June 2010. Physical examination revealed severe anemia and yellow sclera, but no lymphadenopathy, hepatomegaly, or splenomegaly. Laboratory studies revealed $1.9 \mathrm{x}$ $10^{9} / \mathrm{L}$ white blood cells ( $40 \%$ neutrophils, $48 \%$ lymphocytes, $1 \%$ eosinophils, and $11 \%$ monocytes), $41.0 \mathrm{~g} / \mathrm{L}$ hemoglobin, $62 \times 10^{9} / \mathrm{L}$ platelets, $4.9 \mu \mathrm{M}$ direct bilirubin (DBIL), $34.0 \mu \mathrm{M}$ indirect bilirubin (IBIL), $31.9 \mathrm{~g} / \mathrm{L}$ albumin (ALB), $124 \mathrm{U} / \mathrm{L}$ lactate dehydrogenase (LDH), and $3.79 \mathrm{mg} / \mathrm{L} \beta 2$-microglobulin ( $\beta 2-\mathrm{MG}$ ). Ultrasonography revealed no enlargement of the lymph nodes. Positron emission tomography (PET) revealed enhanced bone marrow metabolism but no lymphadenopathy, hepatomegaly, or splenomegaly.

Bone marrow biopsy and aspiration were performed. The bone marrow biopsy specimen indicated active hyperplasia in granulocytes, erythrocytes and megakaryocytes, and cellular morphology was normal. Bone marrow aspirate smears also suggested active hyperplasia, with granulocytes $(49.0 \%)$, erythrocytes $(14.5 \%)$, and lymphocytes $(28.0 \%)$. Of these lymphocytes, $4.5 \%$ showed morphological abnormalities: size differences, tailing, and apophysis; oddly shaped nuclei (pitting, twisted, and folded); and nucleoli with coarse chromatin and rich, blue cytoplasm. Bone marrow flow cytometry revealed a monoclonal B-cell phenotype: CD19 ${ }^{+}$ (2.51\%), $\mathrm{kappa}^{+}, \mathrm{FMC7}^{+}, \mathrm{CD} 23^{+}, \mathrm{CD} 10^{-}, \mathrm{CD}^{-}$, and lambda ${ }^{-}$. The bone marrow chromosomes exhibited normal karyotypes. Washed red blood cells were transfused, and prednisone was administered, thereby improving the condition of the patient: white blood cells, $4.7 \times 10^{9} / \mathrm{L}$; hemoglobin, $60 \mathrm{~g} / \mathrm{L}$; platelets, 87x10/L; DBIL, $3.8 \mu \mathrm{M}$; IBIL, $11.1 \mu \mathrm{M}$; and ALB, $36 \mathrm{~g} / \mathrm{L}$.

Laboratory studies in August 2010 found $4.9 \times 10^{9} / \mathrm{L}$ white blood cells, $38.0 \mathrm{~g} / \mathrm{L}$ hemoglobin, $105 \times 10^{9} / \mathrm{L}$ platelets, $6.2 \mu \mathrm{M}$ DBIL, $22.1 \mu \mathrm{M}$ IBIL, $32.8 \mathrm{~g} / \mathrm{L}$ ALB, $372 \mathrm{U} / \mathrm{L} \mathrm{LDH}$, and $4.38 \mathrm{mg} / \mathrm{L} \beta 2-\mathrm{MG}$. Bone marrow aspirate smears revealed active hyperplasia, with granulocytes $(53.0 \%)$, erythrocytes $(1.0 \%)$, and lymphocytes (40.5\%). Of these lymphocytes, $8.5 \%$ exhibited abnormal morphology. A bone marrow biopsy showed lymphocyte aggregation and cell enlargement. Immunohistochemistry revealed that the lymphocytes were $\mathrm{CD} 20^{+}, \mathrm{CD}^{+}$, $\mathrm{Ki}-67^{+}, \mathrm{PAX}-5^{+}, \mathrm{CD} 10^{-}$, and cyclin $\mathrm{D1}^{-}$. A diagnosis of DLBCL was established. The patient received chemotherapy. Washed red blood cells were transfused, and prednisone was administered. Subsequent laboratory studies revealed $5.7 \times 10^{9} / \mathrm{L}$ white blood cells, $98 \mathrm{~g} / \mathrm{L}$ hemoglobin, $171 \times 10^{9} / \mathrm{L}$ platelets, $4.6 \mu \mathrm{M}$ DBIL, and $28.4 \mu \mathrm{M}$ IBIL.

In March 2011, laboratory studies revealed $3.6 \times 10^{9} / \mathrm{L}$ white blood cells, $58 \mathrm{~g} / \mathrm{L}$ hemoglobin, $49 \times 10^{9} / \mathrm{L}$ platelets, $4.2 \mu \mathrm{M}$ DBIL, and $20.6 \mu \mathrm{M}$ IBIL. A bone marrow aspirate smear showed $30.5 \%$ lymphocytes, $8.0 \%$ lymphoma cells, $11.5 \%$ erythrocytes, and $52.5 \%$ 
granulocytes. Chemotherapy with rituximab, cyclophosphamide, doxorubicin, vincristine, and prednisone (R-CHOP) was administered. Subsequent laboratory studies revealed $6.3 \times 10^{9} / \mathrm{L}$ white blood cells, $71 \mathrm{~g} / \mathrm{L}$ hemoglobin, $77 \times 10^{9} / \mathrm{L}$ platelets, $2.5 \mu \mathrm{M}$ DBIL, and $7.6 \mu \mathrm{M}$ IBIL. A bone marrow aspirate smear showed active hyperplasia, with lymphocytes $(28 \%)$, lymphoma cells (13\%), erythrocytes (33\%), and granulocytes (33\%). In April 2011, another cycle of R$\mathrm{CHOP}$ was administered. Since then, the patient has received only Chinese medicine at home and blood transfusions in the hospital. He remains alive at the time of writing (January 2014).

\section{DISCUSSION}

The secondary involvement of malignant lymphomas in the bone marrow is common, whereas PBM DLBCL is rare. PBM DLBCL accounts for $1.16 \%$ of lymphomas and $2.65 \%$ of DLBCLs. The following criteria (Chang et al., 2011) are used in diagnosing PBM DLBCL: 1) pathologically confirmed bone marrow involvement with DLBCL; 2) pan-B-cell marker (CD19 or CD20) expression shown via immunohistochemistry or flow cytometry analysis; and 3) absence of lymph node involvement (defined as any lymphadenopathy with a size greater than $1 \mathrm{~cm}$ ) shown via whole-body computed tomography or physical examination. The lymphoma cells in our patient were detected via bone marrow aspiration; the proportion of these cells in aspirates ranged from 4.5 to $13.0 \%$. They were $\mathrm{CD} 19^{+}$and $\mathrm{CD} 20^{+}$as determined via bone marrow flow cytometry and biopsy immunohistochemistry. PET revealed no lymphadenopathy, hepatomegaly, or splenomegaly. PBM DLBCL was the correct diagnosis. Hishizawa et al. (2007) emphasized that PET scanning can be useful in diagnosis. In our study, PET revealed striking disseminated metabolism of the bone marrow, without evidence of hepatic, splenic, or lymph node involvement.

Our patient presented with severe pancytopenia, probable autoimmune hemolytic anemia (AIHA, unexplained hyperbilirubinemia), and erythroid hypoplasia. Most PBM lymphomas are accompanied by pancytopenia or bicytopenia at diagnosis. Cytopenia is thought to be caused by lymphoma cell infiltration and autoimmune destruction (Kagoya et al., 2010). Sumi et al. (2007) reported a PBM DLBCL patient presenting with AIHA and erythroid hypoplasia, both of which subsided after three courses of rituximab. The blood cell count and hyperbilirubinemia of this patient also improved. Our patient had high LDH and $\beta 2-\mathrm{MG}$ levels, resulting in tumor hypermetabolism. Almost all reported cases of PBM DLBCL had high LDH levels and a high IPI (Kajiura et al., 2007). The diagnosis of our patient was based on the diffuse infiltration of large lymphoid cells in the bone marrow, immunophenotype, and PET results, in accordance with most diagnostic criteria in the literature.

Our patient was initially treated with washed red blood cell transfusion and prednisone administration, and later with two cycles of R-CHOP. Blood cell numbers and hyperbilirubinemia improved after therapy. Chang et al. (2011) described 4 PBM DLBCL patients who received rituximab-containing regimens [R-CHOP for 3 patients, R-COP (no doxorubicin) for 1] and achieved complete remission. Strauchen (2003) reported 2 PBM DLBCL patients who showed improvement after chemotherapy with CHOP. Patients with bone marrow DLBCL have a poor prognosis. Previous studies described a patient with PBM DLBCL who survived only 3 months (Yoshida et al., 2002) and 3 patients who survived less than 9 months (Alvares et al., 2004). Kajiura et al. (2007) found that the mean survival duration of patients with PBM DLBCL was 14.9 months. Against this dismal background, emerging treatment modalities, targeted therapies, and stem cell transplantation techniques appear promising. A patient who 
underwent six R-CHOP courses followed by high-dose chemotherapy combined with autologous peripheral blood stem cell transplantation remained in complete remission for 12 months after treatment (Hishizawa et al, 2007). Another patient who received R-CHOP chemotherapy achieved complete remission (Kazama et al., 2012). This patient subsequently received autologous stem cell transplant and remained in remission 7 years thereafter. Our patient received two cycles of R-CHOP; although he did not fully recover, he remained alive at the time of writing (January 2014) by chemotherapy and undergoing blood transfusion. Collectively, these findings suggest that individual differences affect treatment outcome. About treatment and prognosis, rituximab therapy is the most effective. The IPI score for PBM DLBCL has remained the same. R-CHOP and autologous stem cell transplantation are recommended treatments for PBM DLBCL.

Kajiura et al. (2007) consider PBM DLBCL a unique entity because the neoplastic cells proliferate mainly in the bone marrow. The origin of bone marrow lymphoma is unclear. Although not observed in isolated marrow DLBCL, complex chromosomal rearrangements typically characterize DLBCLs. Rearrangements involving 1q21-1q23 are associated with poor prognosis (Dorkeld et al., 1999), and trisomy 7q is associated with transformation in nearly $30 \%$ of DLBCL cases. Translocations involving the $3 \mathrm{q} 27$ locus have been reported in 15 to $40 \%$ of DLBCL cases; $1,2 \mathrm{p}^{-}, 6 \mathrm{q}^{-}$, and $14 \mathrm{q}^{+}$chromosomal rearrangements are commonly identified in various lymphomas (Alvares et al., 2004). The bone marrow chromosomes of our patient had normal karyotypes ( $\mathrm{G}$ banding staining). Fluorescence in situ hybridization may increase the detection rate of abnormal karyotypes. The availability of novel cytogenetic data may further elucidate the pathogenic processes involved in DLBCL and help define molecular subgroups of prognosis.

In summary, PBM DLBCL is a distinct subtype of lymphoma characterized by pancytopenia and the absence of lymphadenopathy. It is an advanced disease associated with poor prognosis, but is potentially curable with aggressive treatments, including rituximab-based therapies and stem cell transplantation.

\section{REFERENCES}

Alvares CL, Matutes E and Scully MA (2004). Isolated bone marrow involvement in diffuse large B cell lymphoma: a report of three cases with review of morphological immunophenotypic and cytogenetic findings. Leuk. Lymphoma. 45: 769-775.

Chang H, Hung YS, Lin TL, Wang PN, et al. (2011). Primary bone marrow diffuse large B cell lymphoma: a case series and review. Ann. Hematol. 90: 791-796.

Dorkeld F, Bernheim A, Dessen P and Huret JL (1999). A database on cytogenetics in haematology and oncology. Nucleic Acids Res. 27: 353-354.

Hishizawa M, Okamoto K, Chonabayashi K, Kaneko H, et al (2007). Primary large B-cell lymphoma of the bone marrow. Br. J. Haematol. 136: 351.

Kagoya Y, Sahara N, Matsunaga T, Uekusa T, et al. (2010). A case of primary bone marrow B-cell non-Hodgkin's lymphoma with severe thrombocytopenia: Case report and a review of the literature. Indian J. Hematol. Blood Transfus. 26: 106-108.

Kajiura D, Yamashita Y and Mori N (2007). Diffuse large B-cell lymphoma initially manifesting in the bone marrow. $\mathrm{Am}$. J. Clin. Pathol. 127: 762-769.

Kazama H, Teramura M, Yoshinaga K, Masuda A, et al. (2012). Long-term remission of primary bone marrow diffuse large B-cell lymphoma treated with high-dose chemotherapy rescued by in vivo rituximab-purged autologous stem cells. Case Rep. Med. 2012: article ID 957063.

Strauchen JA (2003). Primary Bone Marrow B-cell Lymphoma: Report of four Cases. Mt. Sinai J. Med. 70: 133-138.

Sumi M, Ichikawa N and Shimizu I (2007). Primary diffuse large B-cell lymphoma of the bone marrow complicated with autoimmune hemolytic anemia and erythroid hypoplasia. Rinsho. Ketsueki. 48: 571-575.

Yoshida K, Kayano H and Akiba M (2002). De novo CD5-positive diffuse by immunohistochemical examination of bone marrow clot sections. Rinsho. Ketsueki. 43: 943-948. 